\title{
Microwave Assisted Ring Opening of Epoxides: A General Route to the Synthesis of 1-Amino- propan-2-ols with Anti Malaria Parasite Activities
}

Aélig Robin, ${ }^{a}$ Fraser Brown, ${ }^{a}$ Noemí Bahamontes-Rosa, ${ }^{b}$ Binghua Wu, ${ }^{c}$ Eric Beitz, ${ }^{c}$ Jürgen F.J. Kun ${ }^{b}$ and Sabine L. Flitsch ${ }^{a}$

${ }^{a}$ Manchester Interdisciplinary Biocentre (MIB), University of Manchester, 131 Princess street, Manchester M1 7ND, UK

${ }^{\mathrm{b}}$ Institute for Tropical Medicine, Department of Parasitology, University of Tübingen, Wilhelmstr. 27, 72074 Tübingen, Germany

${ }^{\mathrm{c}}$ University of Kiel, Department of Pharmaceutical Chemistry, Gutenbergstr. 76, Kiel, Germany

Analytical data for 1d, 1e, 1g, 1i, 1u, 1v, 1w, 2b, 2c, 2d, 2e, 2f, 2g, 3b, 3c...........S2 HPLC analysis for selected compounds $1 \mathbf{b}, \mathbf{1 k}, \mathbf{1 l}, \mathbf{1 p}, \mathbf{1 t}, \mathbf{4 h}, \mathbf{6 c}, \mathbf{8 a}, \mathbf{8 b}, \mathbf{9 a}, \mathbf{9 b} \ldots \ldots . . \mathrm{S} 8$ 
Analytical data for 1d, 1e, 1g, 1i, 1u, 1v, 1w, 2b, 2c, 2d, 2e, 2f, 2g, 3b, 3c.

General. Solvents and reagents were obtained from commercial sources and used as received. Proton and carbon NMR spectra were obtained using a Bruker AC250 or DPX360 instruments. Chemical shifts are reported in parts per million (ppm, $\delta$ ) relative to TMS using $\mathrm{CDCl}_{3}$ as solvent. The coupling constants are reported in Hertz $(\mathrm{Hz})$ using the following abbreviations: $\mathrm{s}=$ singulet, $\mathrm{d}=$ doublet, $\mathrm{t}=$ triplet, $\mathrm{q}=$ quadruplet, qu=quintet and m=multiplet. High Resolution Mass Spectrometry Fast Atom bombardment (HRMS FAB) was performed using a Kratos MS50TC instrument and electrospray (ES-MS) nominal mass spectra were recorded using a Micromass Platform II instrument. All microwave-assisted reactions were carried out in a CEM Discover Microwave Synthesiser with Explorer Carousel.

Benzyl glycidyl ether (for 1a-x), alkyl/aryl glycidyl ether (for 2a-g) or glycidol (for 3a-c) (1 mmol) was dispensed into a microwave tube and dissolved in absolute ethanol (2.5 $\mathrm{mL})$. Amine $(1.5 \mathrm{mmol})$ was added to the tube and placed in the CEM Discover Microwave Synthesiser Explorer Carousel. Each sample was heated at $140^{\circ} \mathrm{C}$ with stirring for 4 minutes ( 80 watts, 50 psi). A period of 5 minutes was allowed for sample cooling. Each sample was concentrated in vacuo and purification was performed if necessary, by using Kugelrohr bulb to bulb distillation apparatus to yield the desired products 1a-x, 2a-g or 3a-c.

1-Benzyloxy-3-piperidin-1-ylpropan-2-ol (1d) Yield 92\%; oil; ${ }^{1} \mathrm{H}$ NMR $\left(\mathrm{CDCl}_{3}\right) ; \delta$ 1.59-1.76 (6H, m, $3 \mathrm{CH}_{2}$ piperidine), 2.49-2.57 (4H, m, $2 \mathrm{NCH}_{2}$ piperidine), 2.70-2.76 $\left(2 \mathrm{H}, \mathrm{m}, \mathrm{NCH}_{2} \mathrm{CH}\right), 3.33-3.42\left(2 \mathrm{H}, \mathrm{m}, \mathrm{CHCH}_{2} \mathrm{O}\right), 3.79\left(1 \mathrm{H}, \mathrm{qu},{ }^{3} \mathrm{~J}=4.6 \mathrm{~Hz}, \mathrm{CHOH}\right)$, $4.47\left(2 \mathrm{H}, \mathrm{s}, \mathrm{OCH}_{2} \mathrm{Ar}\right), 7.15-7.24\left(5 \mathrm{H}, \mathrm{m}, \mathrm{H}_{\mathrm{ar}}\right) ;{ }^{13} \mathrm{C} \mathrm{NMR}\left(\mathrm{CDCl}_{3}\right) \delta 24.2\left(\mathrm{CH}_{2}\right.$ piperidine), 25.9 (2 $\mathrm{CH}_{2}$ piperidine), 54.7 (2 $\mathrm{NCH}_{2}$ piperidine), $61.4\left(\mathrm{NCH}_{2} \mathrm{CH}\right), 66.0$ 
(CHOH), $72.8\left(\mathrm{CHCH}_{2} \mathrm{O}\right), 73.4\left(\mathrm{OCH}_{2} \mathrm{Ar}\right), 127.6\left(\mathrm{CH}_{\mathrm{ar}}\right), 127.7\left(2 \mathrm{CH}_{\mathrm{ar}}\right), 128.4\left(2 \mathrm{CH}_{\mathrm{ar}}\right)$, $138.2\left(C_{\text {ar }}\right) ; m / z$; found $\left(\mathrm{ES}-\mathrm{MS}^{+}\right)[\mathrm{M}+\mathrm{H}]^{+} 250$; found (HRMS FAB) $[\mathrm{M}+\mathrm{H}]^{+} 250.18099$ $\mathrm{C}_{15} \mathrm{H}_{23} \mathrm{NO}_{2}$ requires 250.18070 .

1-Benzyloxy-3-morpholin-4-ylpropan-2-ol (1e) Yield 94\%; oil; ${ }^{1} \mathrm{H}$ NMR $\left(\mathrm{CDCl}_{3}\right) ; \delta$ 2.30-2.36 (4H, m, $2 \mathrm{NCH}_{2}$ morpholine), 2.47-2.55 (2H, m, $\left.\mathrm{NCH}_{2} \mathrm{CH}\right), 3.37-3.41(2 \mathrm{H}, \mathrm{m}$, $\left.\mathrm{CHCH}_{2} \mathrm{O}\right), 3.55-3.62\left(4 \mathrm{H}, \mathrm{m}, 2 \mathrm{OCH}_{2}\right.$ morpholine), $3.84\left(1 \mathrm{H}, \mathrm{qu},{ }^{3} \mathrm{~J}=4.6 \mathrm{~Hz}, \mathrm{CHOH}\right)$, $4.47\left(2 \mathrm{H}, \mathrm{s}, \mathrm{OCH}_{2} \mathrm{Ar}\right), 7.15-7.25\left(5 \mathrm{H}, \mathrm{m}, H_{\mathrm{ar}}\right) ;{ }^{13} \mathrm{C} \mathrm{NMR}\left(\mathrm{CDCl}_{3}\right) \delta 53.3\left(\mathrm{NCH}_{2} \mathrm{CH}\right), 57.7$ (2 $\mathrm{NCH}_{2}$ morpholine), $65.6(\mathrm{CHOH}), 66.5\left(2 \mathrm{OCH}_{2}\right.$ morpholine), $72.1\left(\mathrm{CHCH}_{2} \mathrm{O}\right), 73.1$ $\left(\mathrm{OCH}_{2} \mathrm{Ar}\right), 127.2\left(\mathrm{CH}_{\mathrm{ar}}\right), 127.3\left(2 \mathrm{CH}_{\mathrm{ar}}\right), 128.0\left(2 \mathrm{CH}_{\mathrm{ar}}\right), 137.6\left(\mathrm{C}_{\mathrm{ar}}\right) ; \mathrm{m} / z$; found (ES$\mathrm{MS}^{+}$) $[\mathrm{M}+\mathrm{H}]^{+} 252$; found (HRMS FAB) $[\mathrm{M}+\mathrm{H}]^{+} 252.15970 \mathrm{C}_{14} \mathrm{H}_{21} \mathrm{NO}_{3}$ requires 252.15997 .

1-Allylamino-3-benzyloxypropan-2-ol (1g) Yield 92\%, oil; ${ }^{1} \mathrm{H}$ NMR $\left(\mathrm{CDCl}_{3}\right) ; \delta 2.46$ $2.60\left(2 \mathrm{H}, \mathrm{m}, \mathrm{NCH}_{2} \mathrm{CH}\right), 2.71(1 \mathrm{H}, \mathrm{s}, \mathrm{NH}), 3.12-3.15\left(2 \mathrm{H}, \mathrm{m}, \mathrm{CH}_{2}=\mathrm{CHCH}_{2}\right), 3.32-3.39$ $\left(2 \mathrm{H}, \mathrm{m}, \mathrm{CHCH}_{2} \mathrm{O}\right), 3.76-3.82(1 \mathrm{H}, \mathrm{m}, \mathrm{CHOH}), 4.44\left(2 \mathrm{H}, \mathrm{s}, \mathrm{OCH}_{2} \mathrm{Ar}\right), 5.00-5.10(2 \mathrm{H}, \mathrm{m}$, $\left.\mathrm{CH}_{2}=\mathrm{CHCH}_{2}\right), 5.69-5.83\left(1 \mathrm{H}, \mathrm{m}, \mathrm{CH}_{2}=\mathrm{CHCH}_{2}\right), 7.17-7.24\left(5 \mathrm{H}, \mathrm{m}, \mathrm{H}_{\mathrm{ar}}\right) ;{ }^{13} \mathrm{C} \mathrm{NMR}$ $\left(\mathrm{CDCl}_{3}\right) \delta 51.5\left(\mathrm{CH}_{2}=\mathrm{CHCH}_{2}\right), 52.3\left(\mathrm{NCH}_{2} \mathrm{CH}\right), 68.4(\mathrm{CHOH}), 72.7\left(\mathrm{CHCH}_{2} \mathrm{O}\right), 73.5$ $\left(\mathrm{OCH}_{2} \mathrm{Ar}\right), 116.2\left(\mathrm{CH}_{2}=\mathrm{CHCH}_{2}\right), 127.7\left(\mathrm{CH}_{\mathrm{ar}}\right), 127.8\left(2 \mathrm{CH}_{\mathrm{ar}}\right), 128.5\left(2 \mathrm{CH}_{\mathrm{ar}}\right), 136.6$ $\left(\mathrm{CH}_{2}=\mathrm{CHCH}_{2}\right), 138.1\left(\mathrm{C}_{\mathrm{ar}}\right) ; \mathrm{m} / z$; found $\left(\mathrm{ES}-\mathrm{MS}^{+}\right)[\mathrm{M}+\mathrm{H}]^{+} 222$; found (HRMS FAB) $[\mathrm{M}+\mathrm{H}]^{+} 222.14916 \mathrm{C}_{13} \mathrm{H}_{19} \mathrm{NO}_{2}$ requires 222.14940 .

1-Benzyloxy-3-[(pyridin-2-yl-methyl)amino]propan-2-ol (1i) Yield 47\%, oil, ${ }^{1} \mathrm{H}$ NMR $\left(\mathrm{CDCl}_{3}\right) ; \delta 2.83\left(2 \mathrm{H}, \mathrm{m}, \mathrm{NCH}_{2} \mathrm{CH}\right), 3.51\left(2 \mathrm{H}, \mathrm{d},{ }^{3} \mathrm{~J}=3.9 \mathrm{~Hz}, \mathrm{CHCH}_{2} \mathrm{O}\right), 3.98(3 \mathrm{H}, \mathrm{m}$, $\left.\mathrm{CHOH}, \mathrm{CH}_{2} \mathrm{NH}\right), 4.21(2 \mathrm{H}, \mathrm{s}, \mathrm{NH}, \mathrm{OH}), 4.53\left(2 \mathrm{H}, \mathrm{s}, \mathrm{OCH}_{2} \mathrm{Ar}\right), 7.17(1 \mathrm{H}, \mathrm{m}, \mathrm{CH}$ pyridine), 7.25-7.31 (6H, m, $5 H_{\mathrm{ar}}, \mathrm{CH}$ pyridine), $6.59(1 \mathrm{H}, \mathrm{m}, \mathrm{CH}$ pyridine), $8.52(1 \mathrm{H}$, 
m, $\mathrm{CH}$ pyridine); ${ }^{13} \mathrm{C}$ NMR $\left(\mathrm{CDCl}_{3}\right) \delta 51.7\left(\mathrm{NCH}_{2} \mathrm{CH}\right), 54.1\left(\mathrm{CH}_{2} \mathrm{NH}\right), 68.4(\mathrm{CHOH})$, $72.7\left(\mathrm{CHCH}_{2} \mathrm{O}\right), 73.4\left(\mathrm{OCH}_{2} \mathrm{Ar}\right), 122.3$ ( $\mathrm{CH}$ pyridine), $122.4(\mathrm{CH}$ pyridine $), 127.6-128.3$ (5 $\left.\mathrm{CH}_{\mathrm{ar}}\right), 136.7\left(\mathrm{CH}\right.$ pyridine), $137.9\left(\mathrm{C}_{\mathrm{ar}}\right), 149.2(\mathrm{CH}$ pyridine) $158.0(\mathrm{C}$ pyridine $) ; \mathrm{m} / z$; found $\left(\mathrm{ES}-\mathrm{MS}^{+}\right)[\mathrm{M}+\mathrm{H}]^{+} 273$.

1-Benzyloxy-3-(3,4-dihydroisoquinolin-2(1H)-yl)propan-2-ol (1u) Yield 99\%; oil; ${ }^{1} \mathrm{H}$ NMR $\left(\mathrm{CDCl}_{3}\right) ; \delta$ 2.43-2.78 (6H, m, $\mathrm{NCH}_{2} \mathrm{CH}, 2 \mathrm{CH}_{2}$ dihydroisoquinoline), 3.40-3.44 (2H, m, $\left.\mathrm{CHCH}_{2} \mathrm{O}\right), 3.50\left(\mathrm{~d}, 1 \mathrm{H},{ }^{3} \mathrm{~J}=14.7 \mathrm{~Hz}, \mathrm{CH}_{2}\right.$ dihydroisoquinoline), $3.68(\mathrm{~d}, 1 \mathrm{H}$, ${ }^{3} \mathrm{~J}=14.7 \mathrm{~Hz}, \mathrm{CH}_{2}$ dihydroisoquinoline), 3.89-94 (1H, m, $\left.\mathrm{CHOH}\right), 4.48\left(2 \mathrm{H}, \mathrm{s}, \mathrm{OCH}_{2} \mathrm{Ar}\right)$, 6.88-7.25 (9H, m, $\left.H_{\mathrm{ar}}\right) ;{ }^{13} \mathrm{C}$ NMR $\left(\mathrm{CDCl}_{3}\right) \delta 28.9\left(\mathrm{CH}_{2}\right.$ dihydroisoquinoline), $50.9\left(\mathrm{CH}_{2}\right.$ dihydroisoquinoline), $55.9\left(\mathrm{CH}_{2}\right.$ dihydroisoquinoline), $60.4\left(\mathrm{NCH}_{2} \mathrm{CH}\right), 66.3(\mathrm{CHOH})$, $72.4\left(\mathrm{CHCH}_{2} \mathrm{O}\right), 73.3\left(\mathrm{OCH}_{2} \mathrm{Ar}\right), 125.5-128.5\left(9 \mathrm{CH}_{\mathrm{ar}}\right), 133.9\left(C_{\mathrm{ar}}\right), 134.3\left(C_{\mathrm{ar}}\right), 138.0$ $\left(C_{\mathrm{ar}}\right) ; m / z$; found $\left(\mathrm{ES}-\mathrm{MS}^{+}\right)[\mathrm{M}+\mathrm{H}]^{+} 298$.

1-Benzyloxy-3-(2,3-dihydro-1H-indol-1yl)propan-2-ol (1v) Yield 99\%; oil; ${ }^{1} \mathrm{H}$ NMR $\left(\mathrm{CDCl}_{3}\right) ; \delta 2.80(1 \mathrm{H}, \mathrm{s}, \mathrm{OH}), 3.02\left(2 \mathrm{H}, \mathrm{t},{ }^{3} \mathrm{~J}=7.0 \mathrm{~Hz}, \mathrm{CH}_{2}\right.$ dihydroindole), 3.11-3.78 (6H, m, $\mathrm{CHCH}_{2} \mathrm{OH}, \mathrm{NCH}_{2} \mathrm{CH}, \mathrm{CH}_{2}$ dihydroindole), 4.03-4.13 (1H, m, $\left.\mathrm{CHOH}\right), 4.61(2 \mathrm{H}, \mathrm{s}$, $\left.\mathrm{OCH}_{2} \mathrm{Ar}\right), 6.61\left(1 \mathrm{H}, \mathrm{d},{ }^{3} \mathrm{~J}=8.0 \mathrm{~Hz}, \mathrm{CH}\right.$ dihydroindole $), 6.75\left(1 \mathrm{H}, \mathrm{t},{ }^{3} J=8.0 \mathrm{~Hz}, \mathrm{CH}\right.$ dihydroindole), $7.17\left(2 \mathrm{H}, \mathrm{m}, \mathrm{CH}\right.$ dihydroindole), 7.24-7.51 (5H, m, $\left.H_{\mathrm{ar}}\right) ;{ }^{13} \mathrm{C}$ NMR $\left(\mathrm{CDCl}_{3}\right) \delta 29.2\left(\mathrm{CH}_{2}\right.$ dihydroindole $), 53.9\left(\mathrm{NCH}_{2} \mathrm{CH}\right), 55.2\left(\mathrm{CH}_{2}\right.$ dihydroindole $), 69.3$ $(\mathrm{CHOH}), 72.7\left(\mathrm{CHCH}_{2} \mathrm{O}\right), 74.0\left(\mathrm{OCH}_{2} \mathrm{Ar}\right), 107.6(\mathrm{CH}$ dihydroindole $), 118.5(\mathrm{CH}$ dihydroindole), 124.9-128.9 (5 $\mathrm{CH}_{\mathrm{ar}}, 2 \mathrm{CH}$ dihydroindole), 130.2 ( $\mathrm{C}$ dihydroindole), $138.4\left(C_{\text {ar }}\right), 153.2$ ( $C$ dihydroindole); $m / z$; found $\left(\mathrm{ES}^{\left.-\mathrm{MS}^{+}\right)}[\mathrm{M}+\mathrm{H}]^{+} 284\right.$.

1-Benzyloxy-3-\{[(1-ethylpyrrolidin-2-yl)methyl]amino\}propan-2-ol (1w) Yield 57\%; oil; ${ }^{1} \mathrm{H}$ NMR $\left(\mathrm{CDCl}_{3}\right) ; \delta 1.11\left(3 \mathrm{H}, \mathrm{t},{ }^{3} J=8.7 \mathrm{~Hz}, \mathrm{CH}_{3}\right), 1.64-1.93\left(4 \mathrm{H}, \mathrm{m}, \mathrm{CH}_{2}\right.$ 
pyrrolidine), $2.08-2.28\left(2 \mathrm{H}, \mathrm{m}, \mathrm{CH}_{2}\right.$ pyrrolidine), $2.41-2.89\left(6 \mathrm{H}, \mathrm{m}, \mathrm{CH}_{2} \mathrm{CH}_{3}, \mathrm{NCH}_{2} \mathrm{CH}\right.$, pyrrolidine $\left.\mathrm{CH}_{2} \mathrm{NH}\right), 3.47-3.52\left(2 \mathrm{H}, \mathrm{m}, \mathrm{CHCH}_{2} \mathrm{OH}\right), 3.83-3.90(\mathrm{CHOH}), 4.57(2 \mathrm{H}, \mathrm{s}$, $\left.\mathrm{OCH}_{2} \mathrm{Ar}\right), 7.31-7.36\left(5 \mathrm{H}, \mathrm{m}, \mathrm{H}_{\mathrm{ar}}\right) ;{ }^{13} \mathrm{C}$ NMR $\left(\mathrm{CDCl}_{3}\right) \delta 13.8\left(\mathrm{CH}_{3}\right), 22.7\left(\mathrm{CH}_{2}\right.$ pyrrolidine), $28.9\left(\mathrm{CH}_{2}\right.$ pyrrolidine $), 48.9\left(\mathrm{CH}_{2} \mathrm{CH}_{3}\right), 52.4$ and $52.9\left(\mathrm{NCH}_{2} \mathrm{CH}\right.$ and pyrrolidine $\left.\mathrm{CH}_{2} \mathrm{NH}\right), 53.7\left(\mathrm{CH}_{2}\right.$ pyrrolidine $), 64.0(\mathrm{CH}$ pyrrolidine $), 68.6(\mathrm{CHOH}), 72.8$ $\left(\mathrm{CHCH}_{2} \mathrm{O}\right), 73.3\left(\mathrm{OCH}_{2} \mathrm{Ar}\right), 127.6-128.2\left(5 \mathrm{CH}_{\mathrm{ar}}\right), 138.1\left(\mathrm{C}_{\mathrm{ar}}\right) ; \mathrm{m} / z$; found $\left(\mathrm{ES}-\mathrm{MS}^{+}\right)$ $[\mathrm{M}+\mathrm{H}]^{+} 293$.

1-[Benzyl(methyl)amino]-3-(4-fluorophenoxy)propan-2-ol (2b) Yield 86\%, oil, ${ }^{1} \mathrm{H}$ $\operatorname{NMR}\left(\mathrm{CDCl}_{3}\right) ; \delta 2.31\left(3 \mathrm{H}, \mathrm{s}, \mathrm{NCH}_{3}\right), 2.52-2.71\left(2 \mathrm{H}, \mathrm{m}, \mathrm{NCH} \mathrm{H}_{2} \mathrm{CH}\right), 3.56\left(1 \mathrm{H}, \mathrm{d},{ }^{2} J=13.0\right.$ $\left.\mathrm{Hz}, \mathrm{ArCH}_{2} \mathrm{~N}\right), 3.64(\mathrm{~s}, 1 \mathrm{H}, \mathrm{OH}), 3.71\left(1 \mathrm{H}, \mathrm{d},{ }^{2} J=13.0 \mathrm{~Hz}, \mathrm{ArCH}_{2} \mathrm{~N}\right), 3.95\left(2 \mathrm{H}, \mathrm{d},{ }^{3} J=5.4\right.$ $\left.\mathrm{Hz}, \mathrm{CHCH}_{2} \mathrm{O}\right), 4.10-4.19(1 \mathrm{H}, \mathrm{m}, \mathrm{CHOH}), 6.84-7.02\left(4 \mathrm{H}, \mathrm{m}, H_{\mathrm{ar}}\right), 7.31-7.36(5 \mathrm{H}, \mathrm{m}$, $\left.H_{\text {ar }}\right) ;{ }^{13} \mathrm{C} \mathrm{NMR}\left(\mathrm{CDCl}_{3}\right) \delta 42.6\left(\mathrm{NCH}_{3}\right), 60.0\left(\mathrm{NCH}_{2} \mathrm{CH}\right), 63.0\left(\mathrm{ArCH}_{2} \mathrm{~N}\right), 66.6(\mathrm{CHOH})$, $71.5\left(\mathrm{CHCH}_{2} \mathrm{O}\right), 115.9-116.3\left(4 \mathrm{CH}_{\mathrm{ar}}\right), 127.7-129.9\left(5 \mathrm{CH}_{\mathrm{ar}}\right), 138.7\left(C_{\mathrm{ar}}\right), 155.3\left(C_{\mathrm{ar}}\right)$, $156.2\left(C_{\text {arr }}\right) ; m / z ;$ found $\left(\mathrm{ES}-\mathrm{MS}^{+}\right)[\mathrm{M}+\mathrm{H}]^{+} 290$.

1-[Benzyl(methyl)amino]-3-(4-methoxyphenoxy)propan-2-ol (2c) Yield 88\%, oil, ${ }^{1} \mathrm{H}$ $\left.\operatorname{NMR}\left(\mathrm{CDCl}_{3}\right) ; \delta 2.30(3 \mathrm{H}, \mathrm{s}, \mathrm{NCH})_{3}\right), 2.53-2.72\left(2 \mathrm{H}, \mathrm{m}, \mathrm{NCH} \mathrm{H}_{2} \mathrm{CH}\right), 3.56\left(1 \mathrm{H}, \mathrm{d},{ }^{2} J=13.0\right.$ $\left.\mathrm{Hz}, \mathrm{ArCH}_{2} \mathrm{~N}\right), 3.70\left(1 \mathrm{H}, \mathrm{d},{ }^{2} \mathrm{~J}=13.0 \mathrm{~Hz}, \mathrm{ArCH}_{2} \mathrm{~N}\right), 3.78\left(3 \mathrm{H}, \mathrm{s}, \mathrm{OCH}_{3}\right), 3.95(2 \mathrm{H}, \mathrm{d}$, $\left.{ }^{3} \mathrm{~J}=5.1 \mathrm{~Hz}, \mathrm{CHCH}_{2} \mathrm{O}\right), 4.09-4.18(1 \mathrm{H}, \mathrm{m}, \mathrm{CHOH}), 6.83-6.89\left(5 \mathrm{H}, \mathrm{m}, H_{\mathrm{ar}}\right), 7.30-7.36(4 \mathrm{H}$, $\left.\mathrm{m}, H_{\mathrm{ar}}\right) ;{ }^{13} \mathrm{C} \mathrm{NMR}\left(\mathrm{CDCl}_{3}\right) \delta 42.7\left(\mathrm{NCH}_{3}\right), 56.1\left(\mathrm{OCH}_{3}\right), 60.2\left(\mathrm{NCH}_{2} \mathrm{CH}\right), 63.0$ $\left(\mathrm{ArCH}_{2} \mathrm{~N}\right), 66.8(\mathrm{CHOH}), 71.4\left(\mathrm{CHCH}_{2} \mathrm{O}\right), 115.0\left(2 \mathrm{CH}_{\mathrm{ar}}\right), 116.0\left(2 \mathrm{CH}_{\mathrm{ar}}\right), 127.7-129.5$ (5 $\left.\mathrm{CH}_{\mathrm{ar}}\right), 138.7\left(C_{\mathrm{ar}}\right), 153.4\left(C_{\mathrm{ar}}\right), 154.4\left(C_{\mathrm{ar}}\right) ; \mathrm{m} / z$; found $\left(\mathrm{ES}-\mathrm{MS}^{+}\right)[\mathrm{M}+\mathrm{H}]^{+} 302$.

$N$-(2-\{3-[Benzyl(methyl)amino]-2-hydroxypropoxy\}phenyl)acetamide $\quad$ (2d) Yield 99\%, oil, ${ }^{1} \mathrm{H} \mathrm{NMR}\left(\mathrm{CDCl}_{3}\right) ; \delta 2.10\left(3 \mathrm{H}, \mathrm{s}, \mathrm{COCH}_{3}\right), 2.31\left(3 \mathrm{H}, \mathrm{s}, \mathrm{NCH}_{3}\right), 2.45-2.71(2 \mathrm{H}$, 
m, $\left.\mathrm{NCH}_{2} \mathrm{CH}\right), 3.56\left(1 \mathrm{H}, \mathrm{d},{ }^{2} J=13.0 \mathrm{~Hz}, \operatorname{ArCH}_{2} \mathrm{~N}\right), 3.68\left(1 \mathrm{H}, \mathrm{d},{ }^{2} \mathrm{~J}=13.0 \mathrm{~Hz}, \mathrm{ArCH}_{2} \mathrm{~N}\right)$, 3.85-4.05 (2H, m, $\left.\mathrm{CHCH}_{2} \mathrm{O}\right), 4.09-4.16(1 \mathrm{H}, \mathrm{m}, \mathrm{CHOH}), 6.81-7.01\left(3 \mathrm{H}, \mathrm{m}, H_{\mathrm{ar}}\right), 7.28-$ $7.32\left(5 \mathrm{H}, \mathrm{m}, H_{\mathrm{ar}}\right), 8.26\left(1 \mathrm{H}, \mathrm{d},{ }^{3} J=7.5 \mathrm{~Hz}, H_{\mathrm{ar}}\right), 8.46(1 \mathrm{H}, \mathrm{s}, \mathrm{N} H) ;{ }^{13} \mathrm{C} \mathrm{NMR}\left(\mathrm{CDCl}_{3}\right) \delta$ $24.3\left(\mathrm{COCH}_{3}\right), 42.0\left(\mathrm{NCH}_{3}\right), 58.5\left(\mathrm{NCH}_{2} \mathrm{CH}\right), 62.2\left(\mathrm{ArCH}_{2} \mathrm{~N}\right), 66.0(\mathrm{CHOH}), 72.3$ $\left(\mathrm{CHCH}_{2} \mathrm{O}\right), 113.5\left(\mathrm{CH}_{\mathrm{ar}}\right), 120.5\left(\mathrm{CH}_{\mathrm{ar}}\right), 121.8\left(\mathrm{CH}_{\mathrm{ar}}\right), 123.6\left(\mathrm{CH}_{\mathrm{ar}}\right), 127.3-128.9\left(5 \mathrm{CH}_{\mathrm{ar}}\right.$, $\left.C_{\text {ar }}\right), 137.1\left(C_{\text {ar }}\right), 147.3\left(C_{\text {ar }}\right), 168.8(C O) ; m / z$; found $\left(\mathrm{ES}^{-M^{+}}\right)[\mathrm{M}+\mathrm{H}]^{+} 329$.

1-[Benzyl(methyl)amino]-3-(2-phenyllphenoxy)propan-2-ol (2e) Yield 99\%, oil, ${ }^{1} \mathrm{H}$ NMR $\left(\mathrm{CDCl}_{3}\right) ; \delta 2.29\left(3 \mathrm{H}, \mathrm{s}, \mathrm{NCH}_{3}\right), 2.49-2.68\left(2 \mathrm{H}, \mathrm{m}, \mathrm{NCH}_{2} \mathrm{CH}\right), 3.40(1 \mathrm{H}, \mathrm{s}, \mathrm{OH})$, $3.55\left(1 \mathrm{H}, \mathrm{d},{ }^{2} J=13.2 \mathrm{~Hz}, \operatorname{ArCH}_{2} \mathrm{~N}\right), 3.68\left(1 \mathrm{H}, \mathrm{d},{ }^{2} J=13.2 \mathrm{~Hz}, \operatorname{ArCH}_{2} \mathrm{~N}\right), 4.09(2 \mathrm{H}, \mathrm{s}$, $\left.\mathrm{CHCH}_{2} \mathrm{O}\right), 4.10-4.13(1 \mathrm{H}, \mathrm{m}, \mathrm{CHOH}), 7.07-7.17\left(2 \mathrm{H}, \mathrm{m}, H_{\mathrm{ar}}\right), 7.35-7.43\left(10 \mathrm{H}, \mathrm{m}, H_{\mathrm{ar}}\right)$, 7.64-7.66 $\left(2 \mathrm{H}, \mathrm{m}, H_{\mathrm{ar}}\right) ;{ }^{13} \mathrm{C} \mathrm{NMR}\left(\mathrm{CDCl}_{3}\right) \delta 42.8\left(\mathrm{NCH}_{3}\right), 60.4\left(\mathrm{NCH}_{2} \mathrm{CH}\right), 63.1$ $\left(\mathrm{ArCH}_{2} \mathrm{~N}\right), 66.9(\mathrm{CHOH}), 71.5\left(\mathrm{CHCH}_{2} \mathrm{O}\right), 113.5\left(\mathrm{CH}_{\mathrm{ar}}\right), 121.9\left(\mathrm{CH}_{\mathrm{ar}}\right), 127.4-131.3(12$ $\left.C_{\mathrm{ar}}\right), 131.7\left(C_{\mathrm{ar}}\right), 138.9\left(2 C_{\mathrm{ar}}\right), 156.2\left(C_{\mathrm{ar}}\right) ; m / z$; found $\left(\mathrm{ES}-\mathrm{MS}^{+}\right)[\mathrm{M}+\mathrm{H}]^{+} 348$.

1-[Benzyl(methyl)amino]-3-(2-furylmethoxy)propan-2-ol (2f) Yield 97\%, oil, ${ }^{1} \mathrm{H}$ NMR $\left(\mathrm{CDCl}_{3}\right) ; \delta 2.23\left(3 \mathrm{H}, \mathrm{s}, \mathrm{NCH}_{3}\right), 2.38-2.58\left(2 \mathrm{H}, \mathrm{m}, \mathrm{NCH}_{2} \mathrm{CH}\right), 3.45-3.55(4 \mathrm{H}, \mathrm{m}$, $\left.\mathrm{CHCH}_{2} \mathrm{O}, \mathrm{ArCH}_{2} \mathrm{~N}, \mathrm{OH}\right), 3.64\left(1 \mathrm{H}, \mathrm{d},{ }^{3} \mathrm{~J}=13.2 \mathrm{~Hz}, \mathrm{ArCH}_{2} \mathrm{~N}\right), 3.89-3.94(1 \mathrm{H}, \mathrm{m}, \mathrm{CHOH})$, $4.52\left(2 \mathrm{H}, \mathrm{s}\right.$, furanC $\left.H_{2}\right), 6.34\left(2 \mathrm{H}, \mathrm{d},{ }^{3} \mathrm{~J}=3.0 \mathrm{~Hz}, \mathrm{CH}\right.$ furan $), 7.28-7.42\left(6 \mathrm{H}, \mathrm{m}, 5 H_{\mathrm{ar}}, \mathrm{CH}\right.$ furan); ${ }^{13} \mathrm{C}$ NMR $\left(\mathrm{CDCl}_{3}\right) \delta 42.5\left(\mathrm{NCH}_{3}\right), 60.2\left(\mathrm{NCH}_{2} \mathrm{CH}\right), 62.9\left(\mathrm{ArCH}_{2} \mathrm{~N}\right), 65.7$ (furan $\left.\mathrm{CH}_{2}\right), 67.2(\mathrm{CHOH}), 72.9\left(\mathrm{CHCH}_{2} \mathrm{O}\right), 109.8(\mathrm{CH}$ furan), $110.7(\mathrm{CH}$ furan $)$, 127.6$129.4\left(5 \mathrm{CH}_{\mathrm{ar}}\right), 138.8\left(C_{\mathrm{ar}}\right), 143.2(\mathrm{CH}$ furan $), 156.2(\mathrm{C}$ furan$) ; \mathrm{m} / z$; found $\left(\mathrm{ES}^{-\mathrm{MS}^{+}}\right)$ $[\mathrm{M}+\mathrm{H}]^{+} 276$.

1-[Benzyl(methyl)amino]-3-isopropoxypropan-2-ol (2g) Yield 99\%, oil, ${ }^{1} \mathrm{H}$ NMR $\left(\mathrm{CDCl}_{3}\right) ; \delta 1.15\left(6 \mathrm{H}, \mathrm{s},\left(\mathrm{CH}_{3}\right)_{2} \mathrm{CH}\right), 2.23\left(3 \mathrm{H}, \mathrm{s}, \mathrm{NCH}_{3}\right), 2.22-2.55\left(2 \mathrm{H}, \mathrm{m}, \mathrm{NCH}_{2} \mathrm{CH}\right)$, 
3.37-3.47 (3H, m, $\left.\mathrm{CHCH}_{2} \mathrm{O}, \mathrm{OH}\right), 3.49\left(1 \mathrm{H}, \mathrm{d},{ }^{2} \mathrm{~J}=12.9 \mathrm{~Hz}, \mathrm{ArCH}_{2} \mathrm{~N}\right), 3.53-3.63(1 \mathrm{H}, \mathrm{m}$, $\left.\left(\mathrm{CH}_{3}\right)_{2} \mathrm{CH}\right), 3.63\left(1 \mathrm{H}, \mathrm{d},{ }^{2} \mathrm{~J}=12.9 \mathrm{~Hz}, \mathrm{ArCH}_{2} \mathrm{~N}\right), 3.85-3.93(1 \mathrm{H}, \mathrm{m}, \mathrm{CHOH}), 7.28-7.30$ $\left(5 \mathrm{H}, \mathrm{m}, H_{\mathrm{ar}}\right) ;{ }^{13} \mathrm{C} \mathrm{NMR}\left(\mathrm{CDCl}_{3}\right) \delta 22.5\left(\left(\mathrm{CH}_{3}\right)_{2} \mathrm{CH}\right), 42.6\left(\mathrm{NCH}_{3}\right), 60.4\left(\mathrm{NCH}_{2} \mathrm{CH}\right), 63.0$ $\left(\mathrm{ArCH}_{2} \mathrm{~N}\right), 67.4(\mathrm{CHOH}), 71.1\left(\mathrm{CHCH}_{2} \mathrm{O}\right), 72.5\left(\mathrm{CH}\left(\mathrm{CH}_{3}\right)_{2}\right), 127.4-129.4\left(5 \mathrm{CH}_{\mathrm{ar}}\right)$, $138.9\left(C_{\mathrm{ar}}\right) ; m / z ;$ found $\left(\mathrm{ES}-\mathrm{MS}^{+}\right)[\mathrm{M}+\mathrm{H}]^{+} 238$.

3-(3,4-dihydroisoquinolin-2(1H)-yl)propan-1,2-diol (3b) Yield 99\%, oil, ${ }^{1} \mathrm{H}$ NMR $\left(\mathrm{CDCl}_{3}\right) ; \delta$ 2.43-2.93 (6H, m, $\mathrm{NCH}_{2} \mathrm{CH}, 2 \mathrm{CH}_{2}$ dihydroisoquinoline), 3.44-3.85 (4H, m, $\mathrm{CH}_{2} \mathrm{OH}, \mathrm{CH}_{2}$ dihydroisoquinoline), 3.87-3.91 (1H, m, $\left.\mathrm{CHOH}\right), 7.06-7.20\left(4 \mathrm{H}, \mathrm{m}, H_{\mathrm{ar}}\right)$; ${ }^{13} \mathrm{C}$ NMR $\left(\mathrm{CDCl}_{3}\right) \delta 28.7\left(\mathrm{CH}_{2}\right.$ dihydroisoquinoline $), 51.0\left(\mathrm{CH}_{2}\right.$ dihydroisoquinoline $)$, $56.0\left(\mathrm{CH}_{2}\right.$ dihydroisoquinoline), $60.2\left(\mathrm{NCH}_{2} \mathrm{CH}\right), 64.8\left(\mathrm{CH}_{2} \mathrm{OH}\right), 67.4(C \mathrm{HOH}), 127.5-$ $128.5\left(4 \mathrm{CH}_{\mathrm{ar}}\right), 138.8\left(C_{\mathrm{ar}}\right), 134.0\left(C_{\mathrm{ar}}\right) ; m / z$; found $\left(\mathrm{ES}-\mathrm{MS}^{+}\right)[\mathrm{M}+\mathrm{H}]^{+} 208$.

3-[4-(2-methoxyphenyl)piperazin-1-yl]propan-1,2-diol (3c) Yield 99\%, oil, ${ }^{1} \mathrm{H}$ NMR $\left(\mathrm{CDCl}_{3}\right) ; \delta 2.40-2.43\left(1 \mathrm{H}, \mathrm{dd},{ }^{3} \mathrm{~J}=7.5 \mathrm{~Hz},{ }^{2} \mathrm{~J}=2.4 \mathrm{~Hz}, \mathrm{NCH}_{2} \mathrm{CH}\right), 2.58-2.67(3 \mathrm{H}, \mathrm{m}$, $\mathrm{NCH}_{2} \mathrm{CH}, \mathrm{CH}_{2}$ piperazine), 2.80-2.87 (2H, m, $\mathrm{CH}_{2}$ piperazine), 3.00-3.17 (4H, m, $2 \mathrm{CH}_{2}$ piperazine), $3.51\left(1 \mathrm{H}, \mathrm{dd},{ }^{3} J=6.9 \mathrm{~Hz},{ }^{2} J=2.7 \mathrm{~Hz}, \mathrm{CH}_{2} \mathrm{OH}\right), 3.72\left(1 \mathrm{H}, \mathrm{dd},{ }^{3} J=6.9 \mathrm{~Hz}\right.$, $\left.{ }^{2} J=2.7 \mathrm{~Hz}, \mathrm{CH}_{2} \mathrm{OH}\right), 3.81-3.88\left(4 \mathrm{H}, \mathrm{m}, \mathrm{OCH}_{3}, \mathrm{CHOH}\right), 6.84-6.99\left(4 \mathrm{H}, \mathrm{m}, H_{\mathrm{ar}}\right) ;{ }^{13} \mathrm{C} \mathrm{NMR}$ $\left(\mathrm{CDCl}_{3}\right) \delta 50.1$ (2 $\mathrm{CH}_{2}$ piperazine), $53.2\left(\mathrm{CH}_{2}\right.$ piperazine), $54.9\left(\mathrm{CH}_{2}\right.$ piperazine $), 60.6$ $\left(\mathrm{NCH}_{2} \mathrm{CH}\right), 64.7\left(\mathrm{CH}_{2} \mathrm{OH}\right), 67.0(\mathrm{CHOH}), 77.2\left(\mathrm{OCH}_{3}\right), 126.9-128.7\left(5 \mathrm{CH}_{\mathrm{ar}}\right), 138.3$ $\left(C_{\mathrm{ar}}\right) ; m / z ;$ found $\left(\mathrm{ES}-\mathrm{MS}^{+}\right)[\mathrm{M}+\mathrm{H}]^{+} 267$. 
HPLC analysis for selected compounds $1 \mathrm{~b}, 1 \mathrm{k}, 1 \mathrm{l}, 1 \mathrm{p}, 1 \mathrm{t}, 4 \mathrm{~h}, 6 \mathrm{c}, 8 \mathrm{a}, 8 \mathrm{~b}, 9 \mathrm{a}, 9 \mathrm{~b}$.

Analytical HPLC was performed on an Agilent Series 1100.

\section{HPLC Analysis Table}

\begin{tabular}{|c|c|c|c|c|c|}
\hline \multirow[b]{2}{*}{ Compd } & \multicolumn{3}{|c|}{ HPLC Method A (Normal Phase) } & \multicolumn{2}{|c|}{$\begin{array}{l}\text { HPLC Method B (Reverse } \\
\text { Phase) }^{\mathrm{c}}\end{array}$} \\
\hline & $\begin{array}{l}\text { Purity } \\
(\%)\end{array}$ & $t_{R}(\min )$ & $\begin{array}{c}\text { Isohexane/ } \\
\text { Isopropanol// } \\
\text { Diethylamine }\end{array}$ & Purity (\%) & $t_{R}(\min )$ \\
\hline$( \pm) \mathbf{1 b}$ & 98 & 19.36 and 29.60 & $80 / 20 / 0^{\mathrm{a}}$ & 95 & 7.04 \\
\hline$( \pm) 1 \mathrm{k}$ & 96 & 11.51 and 15.02 & $90 / 10 / 0^{\mathrm{a}}$ & 95 & 7.39 \\
\hline$( \pm) 11$ & 99 & 14.15 and 16.7 & $90 / 10 / 0^{\mathrm{a}}$ & 97 & 7.18 \\
\hline$( \pm) 1 p$ & 95 & 12.57 and 17.02 & $90 / 10 / 0^{\mathrm{a}}$ & 98 & 6.57 \\
\hline$( \pm) 1 t$ & 97 & 29.29 and 32.08 & $90 / 8 / 2^{b}$ & 92 & 6.76 \\
\hline$( \pm) 4 h$ & 98 & 6.17 and 8.81 & $80 / 20 / 0^{\mathrm{a}}$ & 86 & 5.59 \\
\hline$( \pm) 6 c$ & 95 & 9.77 and 17.54 & $98 / 2 / 0^{\mathrm{a}}$ & 93 & 5.64 \\
\hline
\end{tabular}

${ }^{\mathrm{a}}$ Chiralcel OD-H $(5 \mu \mathrm{m}, 250 \times 4.6 \mathrm{~mm}, 1 \mathrm{~mL} / \mathrm{min})$

${ }^{\mathrm{b}}$ Chiracel OJ-H $(5 \mu \mathrm{m}, 250 \times 4.6 \mathrm{~mm}, 1 \mathrm{~mL} / \mathrm{min})$

${ }^{\mathrm{c}}$ Ascentis C8 $(5 \mu \mathrm{m}, 150 \times 4.6 \mathrm{~mm}$, gradient $10-90 \%(0.1 \% \mathrm{TFA})$ acetonitrile in water, $1 \mathrm{~mL} / \mathrm{min})$

\section{HPLC analysis for determination of ee of $8 a, 8 b, 9 a$ and $9 b$.}

\begin{tabular}{|c|c|c|c|c|}
\hline \multirow{2}{*}{ Compd } & \multicolumn{3}{|c|}{ HPLC Method A (Normal Phase) } \\
\cline { 2 - 5 } & $\begin{array}{c}\text { Purity } \\
(\%)\end{array}$ & $\begin{array}{c}\text { ee } \\
(\%)\end{array}$ & $t_{\mathrm{R}}(\min )$ & $\begin{array}{c}\text { Isohexane/ } \\
\text { Isopropanol/ } \\
\text { Diethylamine }\end{array}$ \\
\hline$(\boldsymbol{S})$ 8a & 98 & 99 & 17.02 & $90 / 10^{\mathrm{a}}$ \\
\hline$(\boldsymbol{R}) \mathbf{9 a}$ & 96 & 99 & 12.57 & $90 / 10^{\mathrm{a}}$ \\
\hline$(\boldsymbol{S}) \mathbf{8 b}$ & 95 & 99 & 32.08 & $90 / 8 / 2^{\mathrm{b}}$ \\
\hline$(\boldsymbol{R})$ 9b & 95 & 99 & 29.29 & $90 / 8 / 2^{\mathrm{b}}$ \\
\hline
\end{tabular}

${ }^{a}$ Chiralcel OD-H $(5 \mu \mathrm{m}, 250 \times 4.6 \mathrm{~mm}, 1 \mathrm{~mL} / \mathrm{min})$

${ }^{\mathrm{b}}$ Chiracel OJ-H $(5 \mu \mathrm{m}, 250 \times 4.6 \mathrm{~mm}, 1 \mathrm{~mL} / \mathrm{min})$ 